\title{
Nonsingular Global Fixed-Time Stabilization for Nonlinear Systems
}

\author{
Wei Hu $\mathbb{D}^{1,2}$ Zhangyong Zhou, ${ }^{1,2}$ and Junjun Tang $\mathbb{D}^{1}$ \\ ${ }^{1}$ Stated-Owned Wuhu Machinery Factory, Wuhu 241007, China \\ ${ }^{2}$ Nanjing University of Aeronautics and Astronautics, Nanjing 210000, China \\ Correspondence should be addressed to Wei Hu; huwei9698@126.com
}

Received 10 March 2021; Accepted 8 June 2021; Published 21 June 2021

Academic Editor: Xiaodi Li

Copyright $(02021$ Wei Hu et al. This is an open access article distributed under the Creative Commons Attribution License, which permits unrestricted use, distribution, and reproduction in any medium, provided the original work is properly cited.

Since existing results about fixed-time stabilization are only applied to strict feedback systems, this paper investigates the nonsingular fixed-time stabilization of more general high-order nonlinear systems. Based on a novel concept named coordinate mapping of time domain, a control method is first proposed to transform the nonsingular fixed-time convergence problem into the finite-time convergence problem of a transformed time-varying system. By extending the existing, adding a power integrator technique into the considered time-varying system, a periodic controller is constructed to stabilize the original system in fixed time. The results of simulations verify the effectiveness of the proposed method.

\section{Introduction}

In recent years, more and more attention has been paid to the controller design of high-order nonlinear systems due to its wide application in modeling aerospace craft, rigid robotic systems, and machine systems with underactuation, weak coupling, and instability [1-4]. On the one hand, highorder nonlinear systems are more general forms of strict feedback nonlinear systems, which have been widely studied. More practical systems can be described, including rigid robots, aerospace vehicles, and hydraulic systems. On the other hand, the systems cannot be linearized at the origin or cannot be controlled after linearization when the power of the high-order nonlinear system is not 1 , which makes it difficult to control [2].

For high-order nonlinear system, the finite-time stabilization is studied from different perspectives in [1-6]. However, the convergence time is decided by the initial state of the system in recent achievements. That is to say, the convergence time cannot be prespecified, since the state can be initialed at any point. Besides, when the initial state of the system tends to infinity, the time tends to infinity as well.

The concept of fixed-time stabilization is proposed in [7]. As a special finite-time stabilization method, it requires that the convergence time of the system be bounded and the upper bound be independent of the initial state, which can be set in advance. A primary method for fixed-time stabilization is proposed in [8], which successfully solved the problem of fixed-time stabilization for linear systems with only matching uncertainties. Most of the existing literature is limited to second-order linear systems [9-12]; only [13-15] have studied the fixed-time stabilization for high-order nonlinear systems. Based on the implicit Lyapunov function method, the fixed-time stability of high-order integrators is analyzed in [13]. A nonsmoothed controller is constructed by using the recursive design method to solve the fixed-time stabilization problem of high-order nonlinear interconnected systems [14]. The fixed-time stabilization of strict feedback nonlinear systems with only matching uncertainties is achieved by ingenious state transformation [15]. It should be pointed out that all the systems in [8-15] are merely special forms of higher-order systems. Therefore, it is of great significance to study the fixed-time stabilization of more general high-order nonlinear systems.

Based on the above, the nonsingular global fixed-time stabilization of high-order nonlinear systems is proposed. The main difficulty lies in the design complexity caused by various power terms. Particularly, this issue would be 
intensified if we adopt traditional double-power-term law. The obstacle is partially avoided in this work by using the time-domain mapping, with which the nonsingular fixedtime stabilization problem of the original system is transformed into the finite-time stabilization problem of the corresponding time-varying system; by using the power integration method, the finite-time stabilization problem of the time-varying system is realized. The main innovations are summarized as follows:

(1) A control method based on time-domain mapping is proposed, which transforms the nonsingular fixedtime stabilization problem of the original system into the finite-time stabilization problem of the corresponding time-varying system and provides a new idea for the design of the fixed-time convergence control law. Compared with the traditional doublepower-reaching law, the proposed method is designed with a single-power-reaching law, which is more effective and simpler.

(2) In essence, the fixed-time stabilization can be regarded as the optimal control with fixed terminal time, and the design of its control law is easy to produce singularity, while the control law of the proposed method is nonsingular.

(3) The existing method can only solve the problem of fixed-time stabilization for strict feedback systems with only matching uncertainties, while the proposed method can solve the fixed-time stabilization problem of high-order nonlinear systems with unmatched uncertainties. It is noted that the strict feedback systems are special cases of high-order nonlinear systems; the results of this paper greatly extend the research scope of fixed-time stabilization.

\section{Description}

For the convenience of description, we define $\mathbf{R}, \mathbf{R}_{+}$, and $\mathbf{R}^{n}$ as real number, positive real number, and $n$-dimensional real vectors, respectively, define $\mathbf{C}^{i}$ as $i$-order continuous differentiable function, and define $\mathbf{Q}_{\text {odd }}$ as rational number whose numerator and denominator are positive odd integers.

Consider the following high-order nonlinear systems:

$$
\left\{\begin{array}{c}
\dot{x}_{1}=x_{2}^{p_{1}}+f_{1}\left(\overline{\mathbf{x}}_{1}, t\right), \\
\dot{x}_{2}=x_{3}^{p_{2}}+f_{2}\left(\overline{\mathbf{x}}_{2}, t\right), \\
\vdots \\
\dot{x}_{n}=u^{p_{n}}+f_{n}\left(\overline{\mathbf{x}}_{n}, t\right),
\end{array}\right.
$$

where $\mathbf{x}=\left[x_{1}, x_{2}, \ldots, x_{n}\right]^{T} \in \mathbf{R}^{n}$ and $u \in \mathbf{R}$ represent the state of the system and the control input; $\overline{\mathbf{x}}_{\mathbf{i}}$ is defined as $\left[x_{1}, \ldots, x_{i}\right]^{T} \in \mathbf{R}^{i} ; p_{i}$ is a constant, which belongs to $\mathbf{Q}_{\text {odd }}$; $f_{i}: \mathbf{R}^{i} \times[0,+\infty) \longrightarrow \mathbf{R}$ is an uncertain nonlinear function that satisfies $f_{i}(\mathbf{0}, t)=0, \forall t \in[0,+\infty)$. According to the mean value theorem, it means that there exists a function $\gamma_{i j}\left(\overline{\mathbf{x}}_{i}, t\right)$, which makes $f_{i}\left(\overline{\mathbf{x}}_{i}, t\right)=\sum_{j=1}^{i} x_{j} \gamma_{i j}\left(\overline{\mathbf{x}}_{i}, t\right)$, $i=1, \ldots, n$. Furthermore, the following assumptions are given.

Hypothesis 1. There exists a function $\bar{\gamma}_{i}\left(\overline{\mathbf{x}}_{i}\right) \geq 0$, which makes $f_{i}\left(\overline{\mathbf{x}}_{i}, t\right)=\sum_{j=1}^{i} x_{j} \gamma_{i j}\left(\overline{\mathbf{x}}_{i}, t\right), i=1, \ldots, n$.

Hypothesis 2. For $p_{i} \in \mathbf{Q}_{\text {odd }}, i=1, \ldots, n$, in formula (1), there exists a constant $\eta_{i} \in \mathbf{Q}_{\mathrm{odd}}, i=1, \ldots, n+1$, which is larger than 1, and it makes the following formulas hold:

$$
\begin{aligned}
& 0<\frac{1}{\eta_{1}}-\frac{p_{1}}{\eta_{2}} \leq \frac{1}{\eta_{2}}-\frac{p_{2}}{\eta_{3}} \leq \cdots \leq \frac{1}{\eta_{n}}-\frac{p_{n}}{\eta_{n+1}}, \\
& \frac{p_{i}}{\eta_{i+1}} \leq \min \left\{\frac{1}{\eta_{1}}, \frac{1}{\eta_{2}}, \ldots, \frac{1}{\eta_{i-1}}\right\} .
\end{aligned}
$$

To accurately describe the concept of fixed-time stability, we give the following definitions.

Definition 1. If any solution $\mathbf{x}\left(t, \mathbf{x}_{0}\right)$ of formula (1) reaches the origin at a certain finite time $T\left(\mathbf{x}_{0}\right)$ and then remains at the origin, where $T: \mathbf{R}^{n} \mapsto \mathbf{R}_{+} \cup 0$ is called the convergence time function, then the origin can be called globally finitetime stable. If the origin is globally finite-time stable and the convergence time function $T\left(\mathbf{x}_{0}\right)$ is bounded, that is, $\exists T_{\text {max }}>0$, such that $T\left(\mathbf{x}_{0}\right) \leq T_{\text {max }}, \forall \mathbf{x}_{0} \in \mathbf{R}^{n}$, then the origin is globally fixed-time stable.

The goal of the paper is to design a control input for system (1) to make all closed-loop signals globally bounded and the system state converges to the origin in a fixed time. To facilitate the controller design and stability analysis, the following lemma is introduced from $[6,16]$.

Lemma 1. The Lyapunov function $V(t)$ is assumed to meet the following formula:

$$
\frac{\mathrm{d} V(t)}{\mathrm{d} t} \leq-\rho[V(t)]^{v}
$$

where $\rho$ is a constant and $\rho>0$ and $0<v<1$; then, the following formulas can be established: 


$$
\begin{cases}V(t) \leq\left[V^{1-v}\left(t_{0}\right)-(1-v) \rho\left(t-t_{0}\right)\right]^{1 /(1-v)}, & t_{0} \leq t<\frac{t_{0}+V^{1-v}\left(t_{0}\right)}{\rho(1-v)}, \\ V(t)=0, & t \geq t_{0}+\frac{V^{1-v}\left(t_{0}\right)}{\rho(1-v)}\end{cases}
$$

Lemma 2. For any positive real number $x_{i}, i=1, \ldots, n$, $1<b<1$, the following inequality is established:

$$
\left(\left|x_{1}\right|+\cdots+\left|x_{n}\right|\right)^{b} \leq\left|x_{1}\right|^{b}+\cdots+\left|x_{n}\right|^{b} .
$$

When $b=(p / q) \leq 1$ and $p>0$ and $q>0$, we can obtain

$$
\left|x^{b}-y^{b}\right| \leq 2^{1-b}|x-y|^{b} \text {. }
$$

Lemma 3. For any positive real number $m$, $n$, and function $a(x, y)$, the following inequality is established:

$$
\begin{aligned}
\left|a(x, y) x^{m} y^{n}\right| & \leq c(x, y)|x|^{m+n}+\frac{n}{m+n} \\
& \times\left[\frac{m}{(m+n) c(x, y)}\right]^{m / n}|a(x, y)|^{(m+n) / n}|y|^{m+n},
\end{aligned}
$$

where $c(x, y)>0, x \in R, y \in R$.

\section{Design of Nonsingular Fixed-Time Controller}

3.1. Time-Domain Mapping. In this section, the concept of time-domain mapping is proposed for the first time. The problem of nonsingular fixed-time stabilization of system (1) is transformed into the problem of finite-time stabilization of the time-varying system (12), which simplifies the analysis and design process of fixed-time stabilization.

Assume that the upper bound of the convergence time of system (1) is $T$; when $t \in[0, T)$, the following time-domain coordinate mapping is used to extend the finite-time domain to the infinite-time domain:

$$
\tau=\mu+\sigma \tan \left[\frac{t((\pi / 2)+\arctan (\mu / \sigma))}{T-\arctan (\mu / \sigma)}\right] .
$$

The inverse transformation of it is as follows:

$$
t=T \frac{\arctan ((\tau-\mu) / \sigma)+\arctan (\mu / \sigma)}{(\pi / 2)+\arctan (\mu / \sigma)} \triangleq t_{0}(\tau),
$$

where $\mu \in R$ and $\sigma>0$; according to equation (10), obviously there is $t_{0}(\tau):[0,+\infty) \longrightarrow[0, T)$, and find the derivative on the left and right sides of formula; formula (10) can be transformed as follows:

$$
\frac{\mathrm{d} t}{\mathrm{~d} \tau}=\frac{T}{\sigma[(\pi / 2)+\arctan (\mu / \sigma)]} \cdot \frac{1}{1+[(\tau-\mu) / \sigma]} \triangleq \mathrm{K}(\tau) .
$$

When $t \in[0, T)$, multiplying both sides of formula (1) by formula (11) yields

$$
\left\{\begin{array}{l}
\frac{\mathrm{d} x_{1}}{\mathrm{~d} \tau}=\kappa(\tau)\left[x_{2}^{p_{1}}+\tilde{f}_{1}\left(\overline{\mathbf{x}}_{1}, \tau\right)\right] \\
\frac{\mathrm{d} x_{2}}{\mathrm{~d} \tau}=\kappa(\tau)\left[x_{3}^{p_{2}}+\tilde{f}_{2}\left(\overline{\mathbf{x}}_{2}, \tau\right)\right], \\
\vdots \\
\frac{\mathrm{d} x_{n}}{\mathrm{~d} \tau}=\kappa(\tau)\left[u^{p_{n}}+\tilde{f}_{n}\left(\overline{\mathbf{x}}_{n}, \tau\right)\right],
\end{array}\right.
$$

where $\tilde{f}_{i}\left(\overline{\mathbf{x}}_{i}, \tau\right)=f_{i}\left(\overline{\mathbf{x}}_{i}, t\right) \in \mathbf{C}^{1}$; according to Hypothesis 1 , the following inequality holds:

$$
\left|\widetilde{f}_{i}\left(\overline{\mathbf{x}}_{i}, \tau\right)\right| \leq \bar{\gamma}_{i}\left(\overline{\mathbf{x}}_{i}\right)\left(\left|x_{1}\right|+\cdots+\left|x_{i}\right|\right), \quad i=1, \ldots, n .
$$

Remark 1. For the converted time-varying system (12), $\kappa(\tau)$ can be regarded as the time-varying control coefficient of the system. Therefore, to stabilize system (12), the control law must contain unbounded gain terms $1 / \kappa(\tau)$ to compensate for the effectiveness loss of the time-varying control factor $\kappa(\tau)$. This means that if system (12) is asymptotically stabilized in the time domain $\tau$, although system (1) will achieve a fixed-time stabilization in the time domain $t$, the control input tends to infinity at the terminal time. To overcome the singularity of the control law, a natural method is to achieve finite-time stabilization in time domain $\tau$ so that system (1) can achieve fixed-time stabilization at a certain time $t_{f}<T$. As $\tau$ is boundless, the control law is nonsingular.

Remark 2. The coordinate mapping from time domain $\tau$ to $t$ is not limited to the form of equation (10). Some coordinate maps such as exponential function and trigonometric function are also feasible when they satisfy the following conditions: (1) infinitely differentiable and monotonically increasing; (2) $t_{0}(0)=0$ and $\lim _{\tau \longrightarrow+\infty} t_{0}(\tau)=T$. On the other hand, by adjusting $\mu$ and $\sigma$, a relatively smooth and practical control input can be obtained.

3.2. Design of Finite-Time Controller in Time Domain. In this section, a finite-time state feedback controller is designed in time domain $\tau$ for the time-varying system (12). Firstly, the control parameters are selected. According to Hypothesis 2, a constant $v_{i} \in \mathbf{Q}_{\text {odd }}, i=1, \ldots, n$, which is not less than 1 can be chosen to satisfy the following requirements: 


$$
v_{1}+\frac{p_{1}}{\eta_{2}}=v_{2}+\frac{p_{2}}{\eta_{3}}=\cdots=v_{n}+\frac{p_{n}}{\eta_{n+1}} \triangleq \omega
$$

Extending the power integral method to the timevarying system (12), the finite-time controller is designed in time domain $\tau$ recursively. It should be pointed out that $\dot{V}_{i}$ represents the derivative of $V_{i}$ to $\tau$.

\subsubsection{Choosing the $C^{1}$ Positive Definite Lyapunov Function.}

$$
V_{1}\left(x_{1}\right)=\frac{1}{\eta_{1} v_{1}+1} x_{1}^{\eta_{1} v_{1}+1} \triangleq W_{1}\left(x_{1}\right) \text {. }
$$

Calculating derivation of equation (15), the following equation can be obtained according to formula (3):

$$
\begin{aligned}
\dot{V}_{1}\left(x_{1}\right)= & x_{1}^{\eta_{1} \nu_{1}} \kappa(\tau)\left[x_{2}^{p_{1}}+\tilde{f}_{1}\left(\overline{\mathbf{x}}_{1}, \tau\right)\right] \\
\leq & \kappa(\tau) x_{1}^{\eta_{1} \nu_{1}}\left(x_{2}^{p_{1}}-x_{2}^{* p_{1}}\right)+\kappa(\tau) x_{1}^{\eta_{1} \nu_{1}} x_{2}^{* p_{1}} \\
& +\kappa(\tau) x_{1}^{\eta_{1}\left(v_{1}+p_{1} / \eta_{2}\right)} \rho_{1}\left(x_{1}\right),
\end{aligned}
$$

where $\rho_{1}\left(x_{1}\right) \geq x_{1}^{1-\left(p_{1} \eta_{1} / \eta_{2}\right)} \bar{\gamma}_{1}\left(x_{1}\right) \geq 0$ is the $C^{1}$ function, which can be defined as $\rho_{1}\left(x_{1}\right)=\left(1+x_{1}^{2}\right) \bar{\gamma}_{1}\left(x_{1}\right)$.

According to formula (16), the virtual control law $x_{2}^{*}$ is defined as follows:

$$
x_{2}^{* p_{1}}=-x_{1}^{p_{1} \eta_{1} / \eta_{2}}\left[\frac{n}{\mathrm{~K}(\tau)}+\rho_{1} x_{1}\right] \triangleq-\xi_{1}^{p_{1} / \eta_{2}} \beta_{1}^{p_{1}}\left(x_{1}, \tau\right),
$$

where $\xi_{1}=x_{1}^{\eta_{1}}$ and $\beta_{1}\left(x_{1}, \tau\right)=\left[(n / \kappa(\tau))+\rho_{1}\left(x_{1}\right)\right]^{1 / p_{1}}>0$ are $C^{1}$ functions. Substituting equation (17) into equation (16), the following formula can be obtained:

$$
\dot{V}_{1}\left(x_{1}\right) \leq-n x_{1}^{\eta_{1}\left(\nu_{1}+\left(p_{1} / \eta_{2}\right)\right)}+\kappa(\tau) x_{1}^{\eta_{1} v_{1}}\left(x_{2}^{p_{1}}-x_{2}^{* p_{1}}\right) .
$$

Suppose that, in step $k-1$, there exists a positive Lyapunov function, which satisfies the following equation:

$$
V_{k-1}\left(\overline{\mathbf{x}}_{k-1}, \tau\right) \leq 2 \sum_{i=1}^{k-1} \xi_{i}^{\nu_{i}+\left(1 / \eta_{i}\right)}
$$

Define virtual control law and error as follows:

$$
\begin{aligned}
x_{1}^{*} & =0, \xi_{1}=x_{1}^{\eta_{1}}-x_{1}^{* \eta_{1}}, \\
x_{2}^{*} & =-\xi_{1}^{1 / \eta_{2}} \beta_{1}\left(x_{1}, \tau\right), \quad \xi_{2}=x_{2}^{\eta_{2}}-x_{2}^{* \eta_{2}}, \\
& \vdots \quad \vdots \\
x_{k}^{*} & =-\xi_{k-1}^{1 / \eta_{k}} \beta_{k-1}\left(\overline{\mathbf{x}}_{k-1}, \tau\right), \quad \xi_{k}=x_{k}^{\eta_{k}}-x_{k}^{* \eta_{k}},
\end{aligned}
$$

where $\beta_{i}\left(\overline{\mathbf{x}}_{i}, \tau\right)>0$ and $\xi_{i}$ are $C^{1}$ functions. Besides, $i=1, \ldots, k-1$ and

$$
\dot{V}_{k-1}\left(\overline{\mathbf{x}}_{k-1}, \tau\right) \leq-(n-k+2) \sum_{i=1}^{k-1} \xi_{i}^{\omega}+\kappa(\tau) \xi_{k-1}^{v_{k-1}}\left(x_{k}^{p_{k-1}}-x_{k}^{* p_{k-1}}\right) .
$$

As $\xi_{k-1}$ and $\beta_{k-1}\left(\overline{\mathbf{x}}_{k-1}, \tau\right)>0$ are $C^{1}$ functions, $\eta_{k} \geq 1$; it can be easily obtained that $\xi_{k}=x_{k}^{\eta_{k}}+\xi_{k-1} \beta_{k-1}^{\eta_{k}}\left(\overline{\mathbf{x}}_{k-1}, \tau\right)$ is also a $C^{1}$ function.

Designing $C^{0}$ virtual control law $x_{k+1}^{*}$, which makes equations (19) and (21) hold in step $k$, the $C^{1}$ positive Lyapunov function (equation (22)) is considered.

$$
V_{k}\left(\overline{\mathbf{x}}_{k}, \tau\right)=V_{k-1}\left(\overline{\mathbf{x}}_{k-1}, \tau\right)+\int_{x_{k}^{*}}^{x_{k}}\left(s^{\eta_{k}}-x_{k}^{* \eta_{k}}\right)^{\nu_{k}} \mathrm{~d} s .
$$

According to equations (7) and (19) and mean value theorem, it can be achieved that $V_{k}\left(\overline{\mathbf{x}}_{k}, \tau\right) \leq 2 \sum_{i=1}^{k} \xi_{i}^{v_{i}+\left(1 / \eta_{i}\right)}$, which means that equation (19) is right in step $k$.

Defining that $W_{k}\left(\overline{\mathbf{x}}_{k}, \tau\right)=\int_{x_{k}^{*}}^{x_{k}}\left(s^{\eta_{k}}-x_{k}^{* \eta_{k}}\right)^{v_{k}} \mathrm{~d} s$, calculating derivation of $V_{k}\left(\overline{\mathbf{x}}_{k}, \tau\right)$ to ${ }^{\tau} \tau$, equation (23) can be achieved after combining formulas (20) to (22).

$$
\begin{aligned}
\dot{V}_{k}\left(\overline{\mathbf{x}}_{k}, \tau\right) \leq & -(n-k+2) \sum_{i=1}^{k-1} \xi_{i}^{\omega}+\kappa(\tau) \xi_{k-1}^{v_{k-1}}\left(x_{k}^{p_{k-1}}-x_{k}^{* p_{k-1}}\right)+\kappa(\tau) \xi_{k}^{v_{k}}\left(x_{k+1}^{p_{k}}-x_{k+1}^{* p_{k}}\right)+\kappa(\tau) \xi_{k}^{v_{k}} x_{k+1}^{* p_{k}}+\kappa(\tau) \xi_{k}^{v_{k}} \tilde{f}_{k}\left(\overline{\mathbf{x}}_{k}, \tau\right) \\
& +\sum_{i=1}^{k-1} \frac{\partial W_{k}}{\partial x_{i}} \frac{\mathrm{d} x_{i}}{\mathrm{~d} \tau}+\frac{\partial W_{k}}{\partial \tau} .
\end{aligned}
$$

Let us estimate each term on the right side of equation (23); according to Lemma 2, it can be obtained that

$$
\left|x_{k}^{p_{k-1}}-x_{k}^{* p_{k-1}}\right| \leq 2^{1-\left(p_{k-1} / \eta_{k}\right)}\left|x_{k}^{\eta_{k}}-x_{k}^{* \eta_{k}}\right|^{p_{k-1} / \eta_{k}} \leq 2 \xi_{k}^{p_{k-1} / \eta_{k}} .
$$

According to Lemma 3, there exists a $C^{1}$ function $c_{k}\left(\overline{\mathbf{x}}_{k-1}\right) \geq 0$ making the following inequality right:

$$
\left|\kappa(\tau) \xi_{k-1}^{v_{k-1}}\left(x_{k}^{p_{k-1}}-x_{k}^{* p_{k-1}}\right)\right| \leq \frac{1}{4} \xi_{k-1}^{\omega}+\kappa(\tau) c_{k}\left(\overline{\mathbf{x}}_{k-1}\right) \xi_{k}^{\omega} .
$$

For the convenience of narration, the following lemmas are given to estimate the residual terms on the right side of equation (23). The proof ideas of Lemmas 4 and 5 are similar to those of inequalities (17) and (18) in [4]. Readers can refer to [4] for proof. For simplicity, this paper omits the proof.

Lemma 4. There exits $C^{1}$ function $\widetilde{\gamma}_{k}\left(\overline{\mathbf{x}}_{k}, \tau\right) \geq 0, k=2, \ldots, n$, which makes the following inequality hold:

$$
\left|\kappa(\tau) \xi_{k}^{v_{k}} \tilde{f}_{k}\left(\overline{\mathbf{x}}_{k}, \tau\right)\right| \leq \frac{1}{4} \sum_{i=1}^{k-1} \xi_{i}^{\omega}+\kappa(\tau) \tilde{\gamma}_{k}\left(\overline{\mathbf{x}}_{k}, \tau\right) \xi_{k}^{\omega}
$$


Lemma 5. There exists $C^{l}$ function $\tilde{\rho}_{k}\left(\overline{\mathbf{x}}_{k}, \tau\right) \geq 0, k=2$, $\ldots, n$, which makes the following inequality hold:

$$
\left|\sum_{i=1}^{k-1} \frac{\partial W_{k}}{\partial x_{i}} \frac{\mathrm{d} x_{i}}{\mathrm{~d} \tau}\right| \leq \frac{1}{4} \sum_{i=1}^{k-1} \xi_{i}^{\omega}+\kappa(\tau) \widetilde{\rho}_{k}\left(\overline{\mathbf{x}}_{k}, \tau\right) \xi_{k}^{\omega} .
$$

Lemma 6. There exists $C^{l}$ function $\widetilde{\varphi}_{k}\left(\overline{\mathbf{x}}_{k}, \tau\right) \geq 0, \quad \widetilde{\varphi}_{k}$ $\left(\overline{\mathbf{x}}_{k}, \tau\right) \geq 0$, which makes the following inequality hold:

$$
\left|\frac{\partial W_{k}}{\partial \tau}\right| \leq \frac{1}{4} \sum_{i=1}^{k-1} \xi_{i}^{\omega}+\widetilde{\varphi}_{k}\left(\overline{\mathbf{x}}_{k}, \tau\right) \xi_{k}^{\omega}
$$

3.2.2. Demonstration. It is noticed that the time after transformation is only explicitly included in $x_{k}^{* \eta_{k}}$ which belongs to the expression $W_{k}(k=2, \ldots, n)$. So, we use mathematical induction to analyze $\partial x_{k}^{*} \eta_{k} / \partial \tau$.
According to $x_{2}^{* \eta_{2}}=-x_{1} \beta_{1}^{\eta_{2}}\left(x_{1}, \tau\right)$, where $\beta_{1}\left(x_{1}, \tau\right)>0$ is a $C^{1}$ function, it can be concluded that the partial derivative about $\tau$ to $x_{2}^{* \eta_{2}}$ is available and that the following inequality can be obtained:

$$
\left|\frac{\partial x_{2}^{* \eta_{2}}}{\partial \tau}\right|=\left|\xi_{1} \frac{\partial \beta_{1}^{\eta_{2}}\left(x_{1}, \tau\right)}{\partial \tau}\right| \leq\left|\xi_{1}\right| \varphi_{1}\left(x_{1}, \tau\right),
$$

where $\varphi_{1}\left(x_{1}, \tau\right) \geq 0$ is a designable $C^{1}$ function.

Assuming that when $k=3, \ldots, n, x_{k-1}^{* \eta_{k-1}}$ is partially differentiable to $\tau$, there exists a $C^{1}$ function $\varphi_{k-2}\left(\overline{\mathbf{x}}_{k-2}, \tau\right)$, which makes the following equality hold:

$$
\left|\frac{\partial x_{k-1}^{*} \eta_{k-1}}{\partial \tau}\right|=\varphi_{k-2}\left(\overline{\mathbf{x}}_{k-2}, \tau\right) \sum_{i=1}^{k-2}\left|\xi_{i}\right| .
$$

It should be noticed that $x_{k}^{* \eta_{k}}=-\xi_{k-1} \beta_{k-1}^{\eta_{k}}\left(\overline{\mathbf{x}}_{k-1}, \tau\right)$, where $\beta_{k-1}\left(\overline{\mathbf{x}}_{k-1}, \tau\right)$ is a $C^{1}$ function and $\xi_{k-1}=x_{k-1}^{\eta_{k-1}-x_{k-1}^{*} \eta_{k-1}}$; therefore, $x_{k}^{*} \eta_{k}$ is partially differentiable to $\tau$, and

$$
\begin{aligned}
\left|\frac{\partial x_{k}^{* \eta_{k}}}{\partial \tau}\right| & \leq\left|\xi_{k-1} \frac{\partial \beta_{k-1}^{\eta_{k}}\left(\overline{\mathbf{x}}_{k-1}, \tau\right)}{\partial \tau}\right|+\beta_{k-1}^{\eta_{k}}\left(\overline{\mathbf{x}}_{k-1}, \tau\right)\left|\frac{\partial x_{k-1}^{* \eta_{k-1}}}{\partial \tau}\right| \\
& \leq\left|\xi_{k-1}\right|\left|\frac{\partial \beta_{k-1}^{\eta_{k}}\left(\overline{\mathbf{x}}_{k-1}, \tau\right)}{\partial \tau}\right|+\beta_{k-1}^{\eta_{k}}\left(\overline{\mathbf{x}}_{k-1}, \tau\right) \varphi_{k-2}\left(\overline{\mathbf{x}}_{k-2}, \tau\right) \sum_{i=1}^{k-2}\left|\xi_{i}\right| \\
& \leq \varphi_{k-1}\left(\overline{\mathbf{x}}_{k-1}, \tau\right) \sum_{i=1}^{k-1}\left|\xi_{i}\right|
\end{aligned}
$$

where $\varphi_{k-1}\left(\overline{\mathbf{x}}_{k-1}, \tau\right) \geq 0$ is a designable $C^{1}$ function.

When $k=2, \ldots, n, W_{k}$ is continuous partial differentiable to $\tau$. According to formula (2), we can also know that $v_{k}+\left(1 / \eta_{k}\right)>\omega$; hence, we have the following:

$$
\begin{aligned}
\left|\frac{\partial W_{k}}{\partial \tau}\right| & =\left|\frac{\partial x_{k}^{*} \eta_{k}}{\partial \tau} \int_{x_{k}^{*}}^{x_{k}} v_{k}\left(s^{\eta_{k}}-x_{k}^{* \eta_{k}}\right)^{\nu_{k}-1} \mathrm{~d} s\right| \\
& \leq 2 v_{k} \varphi_{k-1}\left(\overline{\mathbf{x}}_{k-1}, \tau\right)\left|\xi_{k}\right|^{v_{k}-1+\left(1 / \eta_{k}\right)} \sum_{i=1}^{k-1}\left|\xi_{i}\right| \\
& \leq \frac{1}{4} \sum_{i=1}^{k-1} \xi_{i}^{\omega}+\xi_{k}^{\omega} \widetilde{\varphi}_{k}\left(\overline{\mathbf{x}}_{k}, \tau\right),
\end{aligned}
$$

where $\widetilde{\varphi}_{k}\left(\overline{\mathbf{x}}_{k}, \tau\right) \geq 0$ is a designable $C^{1}$ function.
Substituting formulas (25)-(28) into equation (23), the following inequality can be obtained:

$$
\begin{aligned}
\dot{V}_{k}\left(\overline{\mathbf{x}}_{k}, \tau\right) \leq & -(n-k+1) \sum_{i=1}^{k-1} \xi_{i}^{\omega}+\kappa(\tau) \xi_{k}^{\nu_{k}}\left(x_{k+1}^{p_{k}}-x_{k+1}^{* p_{k}}\right) \\
& +\kappa(\tau) \xi_{k}^{\omega}\left[c_{k}\left(\overline{\mathbf{x}}_{k-1}\right)+\widetilde{\gamma}_{k}\left(\overline{\mathbf{x}}_{k}, \tau\right)+\widetilde{\rho}_{k}\left(\overline{\mathbf{x}}_{k}, \tau\right)+\frac{\widetilde{\varphi}_{k}\left(\overline{\mathbf{x}}_{k}, \tau\right)}{\kappa(\tau)}\right]+\kappa(\tau) \xi_{k}^{\nu_{k}} x_{k+1}^{* p_{k}} .
\end{aligned}
$$



follows:

The $C^{0}$ virtual control law in step $k$ can be designed as

$$
x_{k+1}^{*}=-\xi_{k}^{1 / \eta_{k+1}}\left[\frac{n-k+1+\tilde{\varphi}_{k}\left(\bar{x}_{k}, \tau\right)}{\kappa(\tau)}+c_{k}\left(\bar{x}_{k-1}\right)+\tilde{\gamma}_{\kappa}\left(\bar{x}_{k}, \tau\right)+\tilde{\rho}_{\kappa}\left(\bar{x}_{k}, \tau\right)\right]^{1 / p_{\kappa}} \triangleq-\xi_{k}^{1 / \eta_{k+1}} \beta_{\kappa}\left(\bar{x}_{k}, \tau\right)
$$

where $\beta_{k}\left(\overline{\mathbf{x}}_{k}, \tau\right)>0$ is a $C^{1}$ function. Substituting formula (34) into formula (33), the following inequality can be obtained:

$$
\dot{V}_{k}\left(\overline{\mathbf{x}}_{k}, \tau\right) \leq-(n-k+1) \sum_{i=1}^{k} \xi_{i}^{\omega}+\kappa(\tau) \xi_{k}^{v_{k}}\left(x_{k+1}^{p_{k}}-x_{k+1}^{* p_{k}}\right)
$$

According to the above derivation process, the following $C^{0}$ state feedback controller can be designed in step $n$ :

$$
u=x_{n+1}^{*}=-\xi_{n}^{d_{n}} \beta_{n}(\mathbf{x}, \tau),
$$

which makes $C^{1}$ positive Lyapunov function $V_{n}(\mathbf{x}, \tau)$ meet $V_{n}(\mathbf{x}, \tau) \leq 2 \sum_{i=1}^{n} \xi_{i}^{v_{i}+\left(1 / \eta_{i}\right)}$, and the following formula is established:

$$
\dot{V}_{n}(\mathbf{x}, \tau) \leq-\sum_{i=1}^{n} \xi_{i}^{\omega}
$$

Considering that moment $k$ contains the dynamic open set of origin $\bar{\Omega}=\left\{\mathbf{x} \in \mathbf{R}^{n}|| \xi_{i} \mid<1, i=1, \ldots, n\right\}$, we can choose $\varepsilon>$ 0 , which is small enough to make $\widetilde{\Omega}=\left\{\mathbf{x} \in \mathbf{R}^{n} \mid V_{n}(\mathbf{x}, \tau)<\varepsilon\right\}$ be a subset of $\bar{\Omega}$ in time $k$. From formulas (2) and (14), we can know that $v_{i}+\left(1 / \eta_{i}\right)>\omega$, and combining (6) and (37), the following inequality can be achieved:

$$
\frac{\mathrm{d} V}{\mathrm{~d} \tau} \leq-\frac{V^{\rho}}{2^{\rho}}, \quad \forall \mathbf{x} \in \widetilde{\Omega},
$$

where $\rho \min _{i=1, \ldots, n}\left\{v_{i}+\left(1 / \eta_{i}\right)\right\}=\omega$.

In the time domain $\tau$ and, according to equation (37), the solution trajectories of system (12) will enter the set $\widetilde{\Omega}$ in finite time and the finite time can be marked as $\tau_{s}$. It should be noted that $\tau_{s}$ cannot be calculated but can be obtained online by realtime detection of system errors. Furthermore, by using formula (38) and Lemma 1, the finite convergence time of system (12) in time domain $\tau$ can be estimated as follows:

$$
\tau_{f}=\tau_{s}+\frac{2 n^{1-\rho}}{1-\rho}
$$

Remark 3. From formula (37), we can know that $x$ is bounded, and, according to the state feedback control law (36), it can be known that $u$ is also bounded in the closed interval $\left[0, \tau_{f}\right]$ of time domain; that is to say, the actual control law (36) is not singular.

3.3. Design of Fixed-Time Controller in Time Domain $t$. Transform the mapping in (9) into the following compact form:

$$
\tau=\tau_{0}(t)=\frac{\mu^{2}+\sigma^{2}}{\mu+\cot [t((\pi / 2)+\arctan (\mu / \sigma)) / T]} .
$$

Consider that we have already made controller (36) of system (12) into finite-time stabilization in the last section. Let $t_{f}=t_{0}\left(\tau_{f}\right)$, and if the control law in time domain $t$ is designed as

$$
u(\mathbf{x}, t)=-\xi_{n}^{1 / \eta_{n+1}} \beta_{n}\left(\mathbf{x}, \tau_{0}(t)\right), \quad 0 \leq t<t_{f} .
$$

Then, any solution trajectory of system (1) will reach the origin in finite time.

For the time set $\left[k t_{f}, k t_{f}+t_{f}\right), k=1,2, \ldots$ in time domain $t$, assume that the following time-domain coordinate mapping is adopted:

$$
t=t_{k}(\tau)=t_{0}(\tau)+k t_{f} .
$$

The control law can be designed as follows:

$$
u(\mathbf{x}, t)=-\xi_{n}^{1 / \eta_{n+1}} \beta_{n}\left(\mathbf{x}, \tau_{0}\left(t-k t_{f}\right)\right), \quad k t_{f} \leq t<k t_{f}+t_{f} .
$$

The solution trajectory of system (1) will always remain at the origin.

In conclusion, the fixed-time stabilization control law in time domain $t$ can be designed as

$$
u(\mathbf{x}, t)=-\xi_{n}^{1 / \eta_{n+1}} \beta_{n}\left(\mathbf{x}, \tau_{0}\left(\bmod \left(t, t_{f}\right)\right)\right),
$$

where $\bmod \left(t, t_{f}\right)$ denotes modular operation, the result of which is the remainder obtained by $t$ dividing $t_{f}$.

Remark 4. The proposed control scheme provides a novel perspective of fixed-time stabilization. Compared with the traditional method composed of high-power and low-power terms [8-11], there exist only low-power terms in (44), which simplifies the design and analysis process to some degree. Besides, it is worth noticing that the setting times of [8-11, 17] depend on the control parameter, e.g., control gains and power terms. However, the setting time in this work can be directly specified in advance.

\section{Simulation Experiment}

4.1. System Scheme. To verify the effectiveness of the proposed control law, a practical example simulation is used to compare the proposed control method with recent literature.

To the best of our knowledge, existing fixed-time results consider at most so-called normal form systems [15], which is the trivial case of the high-order systems. Therefore, consider the following single link manipulator system: 


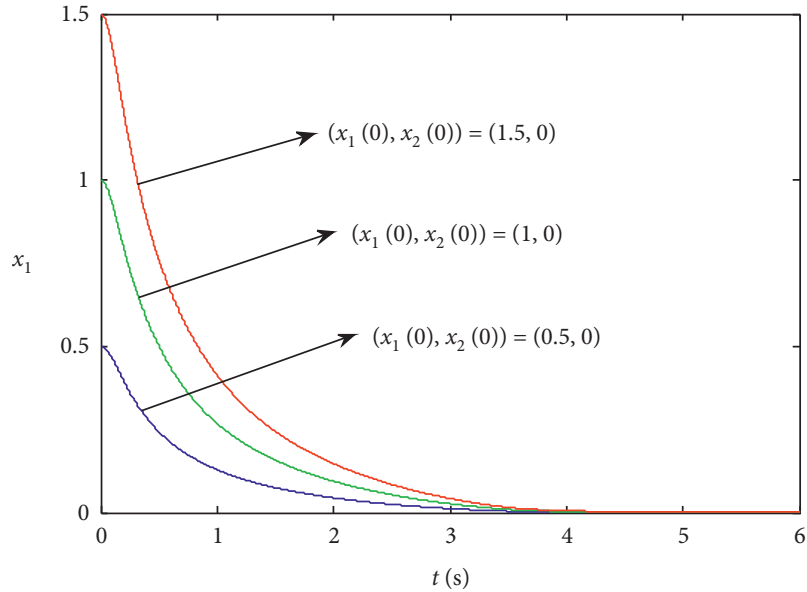

(a)

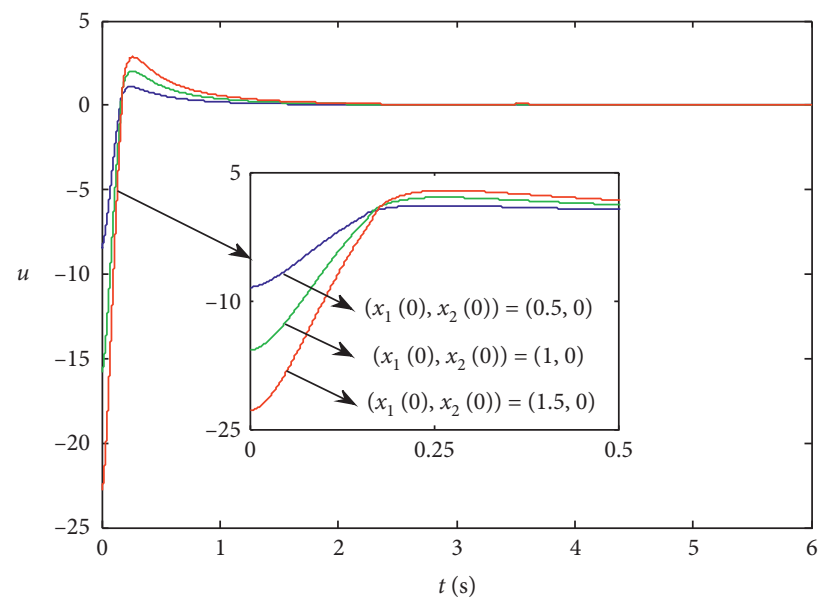

(b)

Figure 1: Response of system (46) to control law (44). (a) Equivalent angular displacement $x_{1}$. (b) Control input $u$.

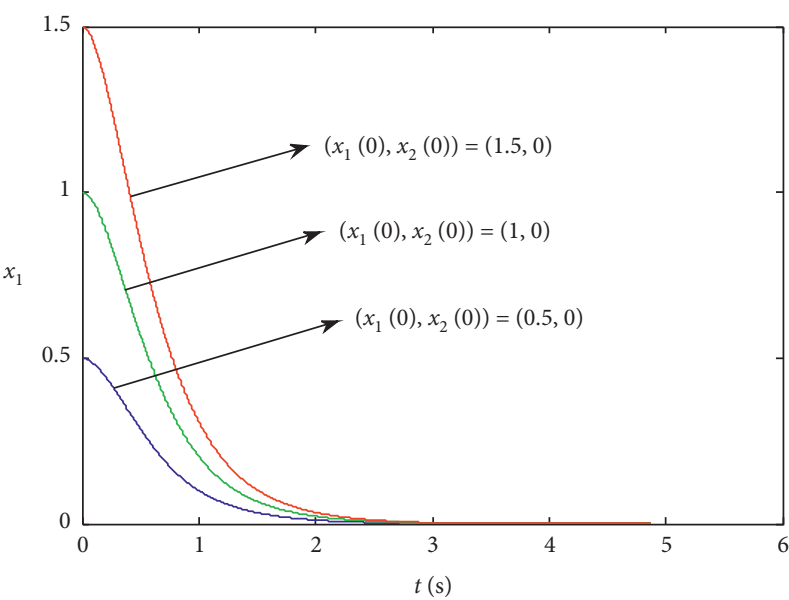

(a)

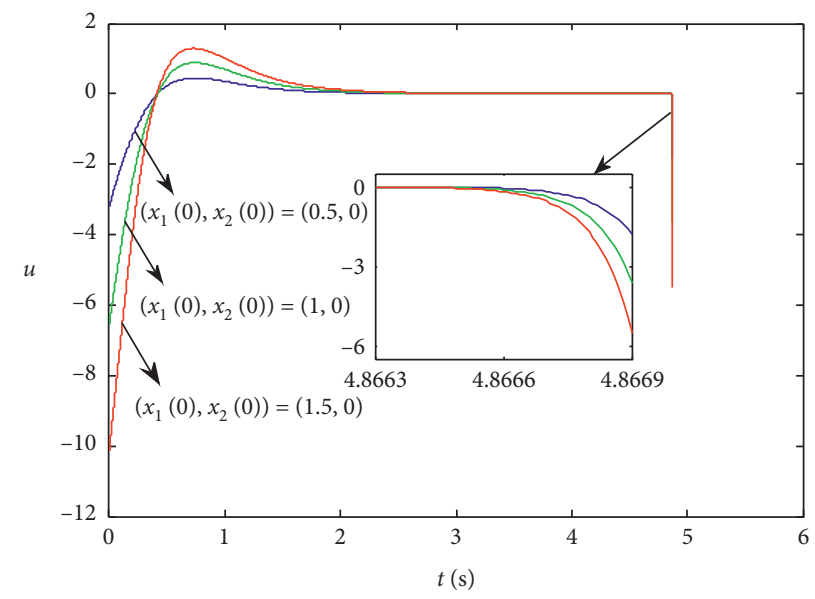

(b)

Figure 2: Response of system (46) to control law in [15]. (a) Equivalent angular displacement $x_{1}$. (b) Control input $u$.

$$
m l \ddot{\varphi}+f l \dot{\varphi}+m g \sin \varphi=u,
$$

where $\varphi \in(-(\pi / 2),(\pi / 2))$ is the rotation angular displacement, $u$ is the external force acting on the manipulator, $m$ is the mass of the manipulator, $l$ is the distance from the center of mass of the manipulator to the rotating shaft, $f$ is the unknown friction coefficient whose upper bound is known, and $g$ is the acceleration of gravity. Assuming that the equivalent angular displacement is $x_{1}=m l \varphi$ and the equivalent angular acceleration is $x_{2}=m l \dot{\varphi}$, equation (45) can be written in the following form:

$$
\left\{\begin{array}{l}
\dot{x}_{1}=x_{2}, \\
\dot{x}_{2}=u-m g \sin \left(\frac{x_{1}}{m l}\right)-\frac{f x_{2}}{m} .
\end{array}\right.
$$

In the above formula, all physical quantities are in $\mathrm{Si}$ basic units, and the values of parameters in the simulation process are selected as $l=g$ and $m=\bar{f}=1 / g$.
For the fixed-time controller (44) designed in this paper, we can choose the parameters as follows: $\eta_{1}=13 / 11, \eta_{2}=11 / 9, \eta_{3}=9 / 7, \nu_{1}=1, v_{2}=103 / 99$, and $\mu=\sigma=1$. For the controller designed in [15], we can choose corresponding parameters as follows: $\lambda=0.1$, $k_{1}=1$, and $k=\theta=2$.

Considering the value range of $\varphi$, the three following groups of initial conditions are selected in the simulation: $x_{1}(0)=0.5, \quad 1, \quad 1.5$ and $x_{2}(0)=0, \quad 0, \quad 0$. Figures 1(a) and 2(a) show the state changes of system (44) with the control law (46) proposed in this paper and with the control law proposed in [15]. It can be seen that, no matter what the initial conditions are, the said two methods achieve stabilization in a fixed time.

It can be seen from Figures 1(b) and 2(b) that the control input in this paper is nonsingular in the whole process; however, that of [15] diverges at the terminal moment. Therefore, from this point of view, the method proposed in this paper is more acceptable. 


\section{Conclusion}

The problem of fixed-time stabilization for high-order nonlinear systems is studied in the paper. A control method based on time-domain mapping is firstly introduced. Compared with the existing literature, the paper proposes a new idea to realize fixed-time stabilization based on timedomain mapping, which greatly expands the research scope of fixed-time stabilization. Note that all the states should be accessible in this work; an interesting problem is the observer design in the case of partially unknown state, which will be considered in our further work.

\section{Data Availability}

The data used to support the findings of this study are included within the article. The original data ca be obtained from the corresponding author upon request.

\section{Conflicts of Interest}

The authors declare that there are no conflicts of interest regarding the publication of this paper.

\section{Acknowledgments}

The authors would like to acknowledge the Major Science and Technology Projects in Anhui Province (no. 18030901058).

\section{References}

[1] Y. J. Zhou, L. Wang, and C. Y. Sun, "Global asymptotic and finite-time stability for nonlinear systems," ACTA Automatica Sinica, vol. 39, no. 5, pp. 664-672, 2013.

[2] Z.-Y. Sun, M.-M. Yun, and T. Li, "A new approach to fast global finite-time stabilization of high-order nonlinear system," Automatica, vol. 81, no. 5, pp. 455-463, 2017.

[3] X. Huang, W. Lin, and B. Yang, "Global finite-time stabilization of a class of uncertain nonlinear systems," Automatica, vol. 41, no. 5, pp. 881-888, 2005.

[4] J. Back, S. G. Cheong, H. Shim, and J. H. Seo, "Nonsmooth feedback stabilizer for strict-feedback nonlinear systems that may not be linearizable at the origin," Systems \& Control Letters, vol. 56, no. 11-12, pp. 742-752, 2007.

[5] J. Fu, T. Y. Chai, C. Y. Su et al., "Adaptive output tracking control of a class of nonlinear systems," Control Engineering of China, vol. 22, no. 4, pp. 731-736, 2015.

[6] J. Fu, R. Ma, and T. Chai, "Adaptive finite-time stabilization of a class of uncertain nonlinear systems via logic-based switchings," IEEE Transactions on Automatic Control, vol. 62, no. 11, pp. 5998-6003, 2017.

[7] V. Andrieu, L. Praly, and A. Astolfi, "Homogeneous approximation, recursive observer design, and output feedback," SIAM Journal on Control and Optimization, vol. 47, no. 4, pp. 1814-1850, 2008.

[8] A. Polyakov, "Nonlinear feedback design for fixed-time stabilization of linear control systems," IEEE Transactions on Automatic Control, vol. 57, no. 8, pp. 2106-2110, 2012.

[9] Y. Dvir and A. Levant, "Accelerated twisting algorithm," IEEE Transactions on Automatic Control, vol. 60, no. 10, pp. 2803-2807, 2015.
[10] J. P. Mishra, C. J. Li, X. H. Yu et al., "Fixed-time converging terminal surface with non-singular control design for secondorder systems," in Proceedings of IFAC World Congress, pp. 5139-5143, Elsevier, Toulouse, France, July 2017.

[11] B. Tian, Z. Zuo, X. Yan, and H. Wang, "A fixed-time output feedback control scheme for double integrator systems," Automatica, vol. 80, no. 3, pp. 17-24, 2017.

[12] Y. Liu, Y. Zhao, W. Ren, and G. Chen, "Appointed-time consensus: accurate and practical designs," Automatica, vol. 89, no. 3, pp. 425-429, 2018.

[13] A. Polyakov, D. Efimov, and W. Perruquetti, "Finite-time and fixed-time stabilization: implicit Lyapunov function approach," Automatica, vol. 51, no. 1, pp. 332-340, 2015.

[14] C. Hua, Y. Li, and X. Guan, "Finite/fixed-time stabilization for nonlinear interconnected systems with dead-zone input," IEEE Transactions on Automatic Control, vol. 62, no. 5, pp. 2554-2560, 2017.

[15] Y. Song, Y. Wang, J. Holloway, and M. Krstic, “Time-varying feedback for regulation of normal-form nonlinear systems in prescribed finite time," Automatica, vol. 83, no. 7, pp. 243-251, 2017.

[16] J. Wu, D. Yang, X. He, and X. Li, "Finite-time stability for a class of underactuated systems subject to time-varying disturbance," Complexity, vol. 2020, Article ID 8704505, 7 pages, 2020.

[17] J. Hu, G. Sui, X. Lv, and X. Li, "Fixed-time control of delayed neural networks with impulsive perturbations," Nonlinear Analysis: Modelling and Control, vol. 23, no. 6, pp. 904-920, 2018. 